Research Article

\title{
Distribution Network Topology Identification Based on IEC 61850 Logical Nodes
}

\author{
Yu Chen $\mathbb{D}^{1},{ }^{1}$ Lingyan Sun $\mathbb{D}^{1},{ }^{1}$ Zonghui Wang, ${ }^{1}$ and Jinghua Wang ${ }^{2}$ \\ ${ }^{1}$ School of Electrical and Electronic Engineering, Shandong University of Technology, Zibo 255000, China \\ ${ }^{2}$ Shandong Kehui Electric Automation Co., Ltd., Zibo 255000, China \\ Correspondence should be addressed to Yu Chen; chenyu@sdut.edu.cn
}

Received 3 October 2020; Revised 23 November 2020; Accepted 6 February 2021; Published 22 February 2021

Academic Editor: Ting Yang

Copyright $\left({ }_{0} 2021\right.$ Yu Chen et al. This is an open access article distributed under the Creative Commons Attribution License, which permits unrestricted use, distribution, and reproduction in any medium, provided the original work is properly cited.

Distributed control has good real-time performance and can better meet the control requirements of active distribution networks with a large number of distributed generations. Some distributed applications require real-time feeder topology to achieve control. In this paper, the demand for distributed control applications for feeder real-time topology is analyzed. Based on IEC 61850 modeling method, a new cell topology logic node and a new topology slice node are built to express feeder topology. Using the topology information of smart terminal unit (STU) configuration and the current status information of switchgear, based on the depth-first search, the feeder real-time topology identification can be realized, which meets the application requirements of distributed control. The study case verified the effectiveness of the method.

\section{Introduction}

Distributed control has good real-time performance and can better meet the control requirements of active distribution networks with a large number of distributed generations. For distributed applications that need to use feeders or local information to make decisions, a real-time feeder topology is needed to achieve control. Taking distributed feeder automation (FA), which is a typical application of distributed control, as an example, it can realize rapid fault location, isolation, and service restoration of feeders, shorten the power outage time of nonfaulty sections to a few seconds, and improve power supply reliability $[1,2]$.

Distributed FA does not rely on the master station, it only needs the communication between the distribution STUs to make decisions, so it can effectively improve the processing speed of distribution network fault. The implementation of the distributed FA function needs to know the current real-time topology of the feeder [3, 4], especially the location of the tie switch when the power supply is restored. However, due to the operation of fault isolation, load transfer, and network optimization, the location of the tie switch may change. Therefore, for distributed feeder automation, how to identify the real-time topology of the distribution network is the key problem to be solved $[5,6]$.

The real-time topology of the distribution network is determined by its static topology combined with the on-off state of switches on the feeder. The purpose of topology identification is to form the current feeder real-time topology according to the switch real-time state for the operation control of the distribution network [7, 8]. At present, there are two modes of distribution network topology processing; one is the master station processing mode and the other is the distributed processing mode. Some DMS functions need the real-time topology of the distribution network, such as centralized FA. The topology identification algorithm mainly includes the tree search method and adjacency matrix method $[9,10]$. In the centralized FA mode, the STU on the feeder does not store the feeder topology information. The static topology of feeders is stored in the master station, and the real-time topology is obtained by the topology recognition algorithm. When the topology of distribution lines changes, the complete topology of feeders will be updated in the master station. In this way, the topology information is complete, but it can only be used for centralized processing applications [11]. In order to 
complete the control, the distributed control application needs the real-time topology of the feeder, but it generally does not need the complete topology of the feeder. It only needs the topology information in the control domain [12]. In order to meet the needs of distributed control topology information, the research of real-time distribution line topology identification has been carried out in the literature. For the expression and configuration of topology, Zhu and Cong' team configure the adjacency relationship of adjacent switches on feeders based on the user-defined format to realize the feeder automation function $[13,14]$. Zhu' team describes the distribution network topology based on the Process and Line model of IEC 61850 system configuration language (SCL) and proposes a distributed topology processing method based on Graph Segmentation and realizes the topology identification of distribution network by communication of STUs $[15,16]$. The topology description method based on SCL needs to generate a topology configuration file and store it in the STUs. The real-time topology is obtained by transferring and exchanging the topology configuration file. Except for the topology configuration, other functions are achieved by logical nodes. Fan' team proposes a method, which uses the STU local topology matrix, and realizes topology recognition after exchanging information between STUs [17].

In this paper, the IEC 61850 method is used to model logical node (LN) RTCN and RTPM to express the topology in the feeder. Based on the logical nodes, the topology recognition algorithm is studied to complete the topology search of feeders to meet the application requirements of distributed control applications. Compared with the method of describing distribution network topology based on SCL, the method of describing topology information based on logical nodes, and the method of configuration and acquisition of local topology are consistent with other functional logical nodes, which is more convenient for application and promotion of distributed control application.

\section{Distributed Control Application Topology}

2.1. Distributed Control. Distributed control application deploys functions to STU and uses STU's communication with each other to exchange detection and control information to achieve corresponding functions. Taking distributed FA as an example, after STU on the line switch detects the fault information, they exchange information and decide to isolate the fault section. After the fault section is isolated successfully, the nonfault upstream section is restored by closing the main breaker. If there is a tie switch in the nonfault downstream section of the fault section, it is restored by closing the tie switch.

Taking Figure 1 as an example, CB is the circuit breaker, S1, S2, S3, and S5 are sectional switches, and S4 are tie switches. STU is installed at the main breaker, section switch, and tie switch, and STU communicates through a peer-topeer communication network.

In the application of distributed FA, it is necessary to know the topology relationship of each device. When the fault isolation of distributed FA is completed, the power supply of the nonfault section needs to be restored. The system needs to know the topology of the fault line downstream to determine the appropriate power supply recovery path.

2.2. Static Topology. Network static topology refers to the static adjacent relationship between the distribution equipment and the line. The change of the adjacent relationship between the distribution equipment will lead to the change of the static topology. The construction of distribution lines, such as line extension, switch increase and decrease, and new equipment put into operation, will change the static topology of the network.

2.3. Application Topology. Application topology refers to the real-time adjacent relationship of related devices in the control domain when a specific function is realized. The realtime topology of the feeder is determined by the static topology combined with the switching state of the switch. Taking the distributed FA as an example, the real-time topology generally refers to the real-time connection relationship of the medium-voltage distribution feeder starting from the substation bus, and the endpoint is the load, the opposite substation bus, the distributed generation, and the tie switch. When fault location, isolation, and recovery, it is necessary to know the upstream and downstream connection of real-time STU. In this paper, based on the new logical node to express the static topology of the feeder, combined with the current switch state information, the search algorithm is used to obtain the real-time application topology of the feeder.

The change of switch state will cause the change of application topology, as shown in Figure 1. Before the fault, the connection relationship of the distribution network is CB1-S1-S2-S3 and CB2-S5-S4. After the failure, due to the switch operation, the application topology changes, as shown in Figure 2, and its connection relationship becomes CB1-S1, CB2-S5-S4.

\section{Distribution Network Topology Representation}

3.1. Existing Distribution Network Topology Model. The topological model of the network is the basis of all network analysis applications. The substation configuration description language SCL defined in IEC 61850-6 Ed2.1 can describe the feeder topology, intelligent electronic device (IED) information model, and communication service of the system. The work by Zhu' team [18], IEC 61850-6 Ed2.1, describes the distribution network topology through new Process and Line elements. In the SCL model description of IEC $61850 \mathrm{Ed} 2.1$, the main line and branch line in the distribution network are all represented by the Line element, and the medium-voltage/low voltage distribution substation and switching station in the distribution network are described by Substation. When the network contains both Substation and Line, the process container needs to be used on the upper layer to represent the local network of a system. After the topological model of feeders described by SCL is 


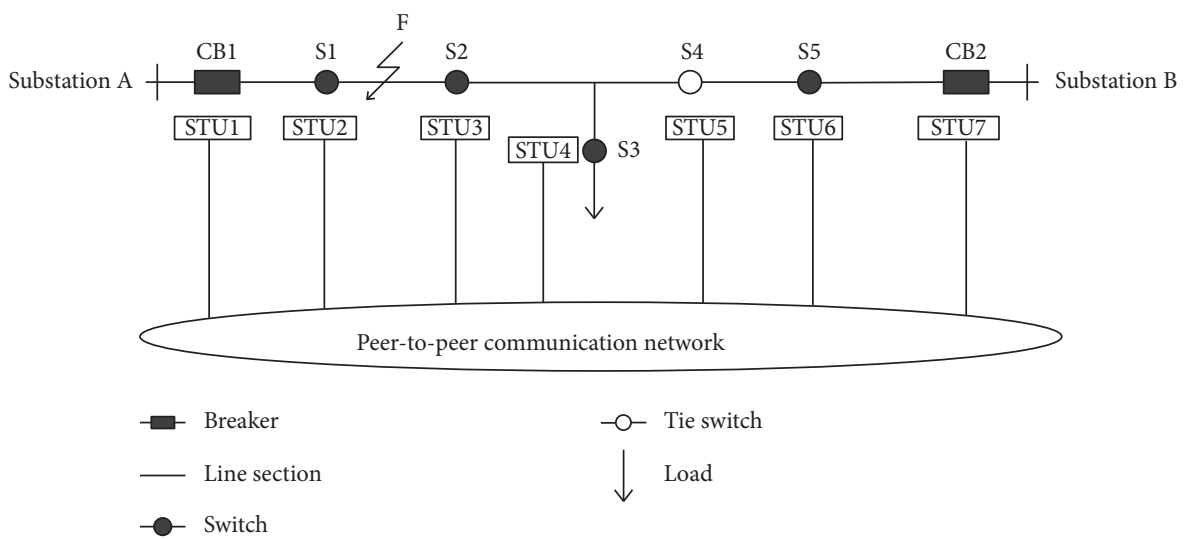

FIgURe 1: Typical distribution lines.

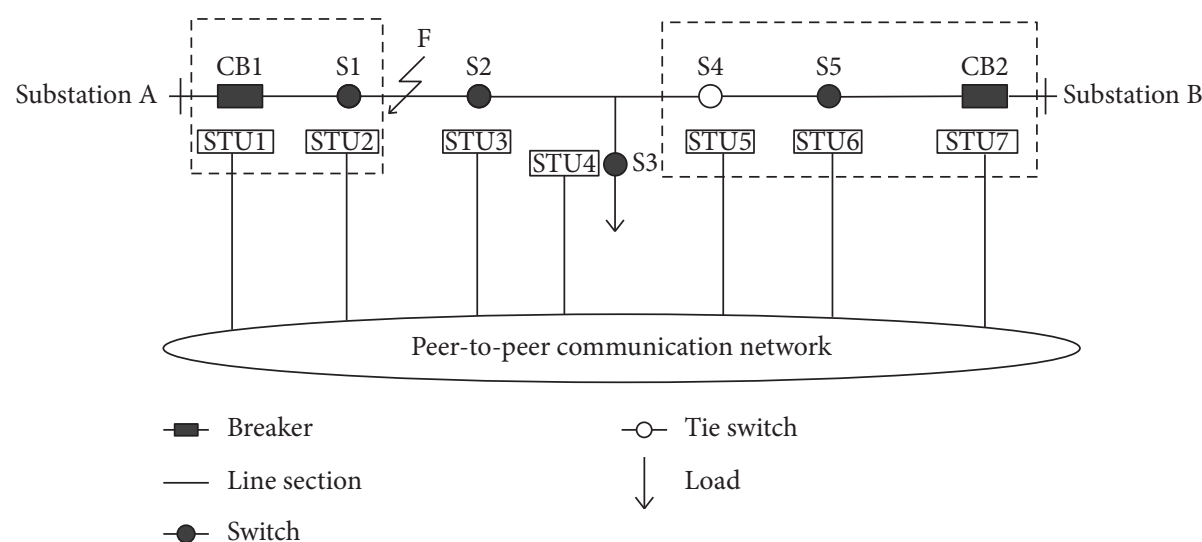

Figure 2: Typical distribution lines after fault.

established, the topological model needs to be configured in the IED of feeders. There are two configuration modes: the first is to divide the complete topology model of the feeder in the master station and then send it to the corresponding IED on the feeder to complete the configuration, the second is to use the SCL file on the IED to describe the local topology, and the IED uploads the master station to form the complete topology of the feeder in the master station.

3.2. Solution of Topology Description Based on Topology Node. For distributed FA applications, STU needs to know the current topology connection relationship to perform fault location and isolation and need to define the complete topology of the feeder to determine the recovery power supply path.

To complete the distributed FA application, the detailed feeder complete topology should be configured in the STU, which needs large storage space and is difficult to deal with when using, so it is necessary to study how to effectively simplify the expression of topology.

When using the topology relationship, distributed FA is mainly concerned with the connection relationship of conductive equipment and does not need the length of wire and other detailed electrical parameters.
In the topological description, we can mode the wire and regard it as a logical connecting line. After the wire is modeled as a logical connecting line, the configuration of the STU can be simplified, and the implementation of distributed FA applications can be better supported.

Based on this, this paper studies the topology description and uses IEC 61850 modeling method to create a new logical node to represent the topology structure.

3.2.1. Cell Topology Logical Node. Based on the modeling rule of IEC 61850, a new logical node of cell topology is built, which is the smallest logical unit of feeder topology. Each cell topology logic node represents the connection relationship between the conductive equipment on the feeder and its adjacent conductive equipment.

In the equipment model in CIM, equipment models are divided into conductors, transformer windings, loads, connectors, equivalent power supplies, regulating equipment, and switches. Referring to CIM's equipment modeling, this paper divides the equipment on the distribution line into the equipment with breaking capacity and the equipment without breaking capacity. The equipment with breaking capacity is uniformly defined as switch type, and the equipment type is also defined for the bus and 
transformer, which do not have breaking capacity but affect the boundary judgment of topology identification.

The smallest unit of the feeder is represented by the logical nodes of cell topology, and the local feeder topology can be represented by multiple logical nodes of cell topology, and then the local topology is formed by the integration of the logical nodes of cell topology. When the network changes, only the data of the cell topology logical node corresponding to the changed device needs to be updated.

3.2.2. Topology Slice Node. Because STU is not installed on every switch on the feeder, a container is needed to store a local area topology of multiple switches. The topology slice node represents the local area topology of feeders. The local area topology is composed of multiple cell topology logical nodes, so the topology slice node contains all the cell topology logical nodes that make up the local area topology. The complete topology of feeders is composed of several local area topologies. When the adjacency relationship of local area topology is expressed clearly, multiple local area topologies can be combined into a complete topology of the feeder.

When describing the topological relationship of feeders, all conductive devices have unique names. In the description of the topology slice, the boundary of the topology slice needs to be determinate. The topological slice is bounded by bus bars, transformers, and tie switches. The cell topology logic node search in the topology slice is limited to the boundary of the topology slice. The topology slice generally corresponds to the control area of the STU.

\section{Topological Logical Node Modeling}

4.1. Cell Topology LN Modeling. According to the requirements of distributed FA application for topology, the cell topology logic node needs to express (1) conductive equipment on the feeder, (2) type of conductive equipment, (3) conductive equipment adjacent to the equipment, and (4) number of adjacent conductive equipment. Based on this, a new cell topology logical node RTCN is created, where $R$ represents that the logical node belongs to the protection related function node group, $T$ is the abbreviation of topology, and $\mathrm{CN}$ represents the connection node. The main data objects of the cell topology logical nodes are shown in Table 1. M/O/C means required/optional/condition required.

The CeName attribute in the cell topology logical node represents the name of the conductive device. PTRType refers to the type of conductive equipment, which can be distinguished according to whether it has breaking capacity. The equipment with breaking capacity on the line is a switch, which is represented by constant 1 in PTRType attribute, while the equipment without breaking capacity is represented by substation bus, switch station bus, and transformer, which is represented by constant 2, 3, and 4 in PTRType attribute. The AdjRTCN attribute represents other cell topology logical nodes connected to the cell topology logical node. In this data object, there can be multiple cell topology logical nodes adjacent to it. Multiple cell topology logical nodes form an array, and the attribute type is a character string. The AdjRTCNNum attribute indicates the number of adjacent cell topology logical nodes.

4.2. Topology Slice LN Modeling. On the basis of the logical node of the cell topology, a new topology slice node (RTPM) is needed to describe the local area topology of the feeder. The topology slice node (RTPM) also belongs to the protection related function node group. The topology slice node needs to express (1) the cell topology logic node covered by this topology slice, (2) other topology slices adjacent to this topology slice node, (3) the number of adjacent topology slices. The data objects of the topology slice node are shown in Table 2.

The function of the topology slice node is to describe the connection relationship between the topology slices and include all the cell topology logical nodes in the topology slice. The following is a description of the main data objects of the topology slice node:

(1) AdjRTPM attribute refers to the adjacency topological slices of this topological slice. All adjacency topological slices form an array, and its attribute type is a string.

(2) The AdjRTPMNum attribute represents the number of adjacent topologies. The attribute type is a numeric constant.

(3) The RTPMCovRTCN attribute represents the cell topology logical nodes covered by this topology slice. All the cell topology logical nodes form an array, and the attribute type is a string.

As shown in Figure 3, the relationship between the cell topology logical node and the topology slice node and the relationship between the local topology stored in the corresponding STU are shown. The cell topology logical nodes RTCN1 and RTCN2 belong to the topology slice RTPM1, and the topology slice RTPM1 is stored in STU1.

Taking the feeder in Figure 3 as an example, the topology of the unit topology logical node and the topology slice node is described as follows:

(1) The AdjRTPM attribute of the topology chip RTPM1 stored in STU1 indicates that the adjacency topology slice node is RTPM2, AdjRTPMNum indicates that the number of adjacency topology slices is 1 , and the RTPMCovRTCN attribute indicates that the RTPM1 contains the unit topology logic nodes RTCN1 and RTCN2. RTPM1 data block diagram is shown in Figure 4.

(2) RTCN3 included in RTPM2 indicates the switch K1 on the line. The AdjRTCN attribute in RTCN3 indicates that the adjacent cell topological are RTCN2, RTCN4, and RTCN6. Its PTRType attribute is the constant 1 , which represents a switch on the line. The CeName attribute indicates that the specific equipment name of the distribution line is $10 \mathrm{kV} \$ * *$ line $\$ \mathrm{~K} 1$. RTCN3 data block diagram is shown in Figure 5. 
TABLE 1: Main data objects of RTCN.

\begin{tabular}{|c|c|c|c|c|}
\hline Attribute name & Attribute types & Explanation & $\mathrm{M} / \mathrm{O} / \mathrm{C}$ & \\
\hline CeName & VISIBLE STRING & Name of conductive equipment & M & \\
\hline PTRType & INS & Type of conductive equipment & M & $\begin{array}{c}\text { 1- Switch } \\
\text { 2- Substation bus } \\
\text { 3- Bus of switching station } \\
\text { 4- Transformer } \\
\text { 5- other }\end{array}$ \\
\hline AdjRTCN & ARRAY[0...Num] of VISIBLE STRING & Adjacency RTCN & M & \\
\hline AdjRTCNNum & INS & Number of adjacent RTCN & M & \\
\hline
\end{tabular}

TABle 2: Main data objects of RTPM.

\begin{tabular}{lccc}
\hline Attribute name & Attribute types & Explanation & M/O/C \\
\hline AdjRTPM & ARRAY[0...Num] of VISIBLE STRING & Adjacency RTPM & M \\
AdjRTPMNum & INS & Number of adjacent RTPM & M \\
RTPMCovRTCN & ARRAY[0...Num] of VISIBLE STRING & RTCN included in RTPM & M \\
\hline
\end{tabular}

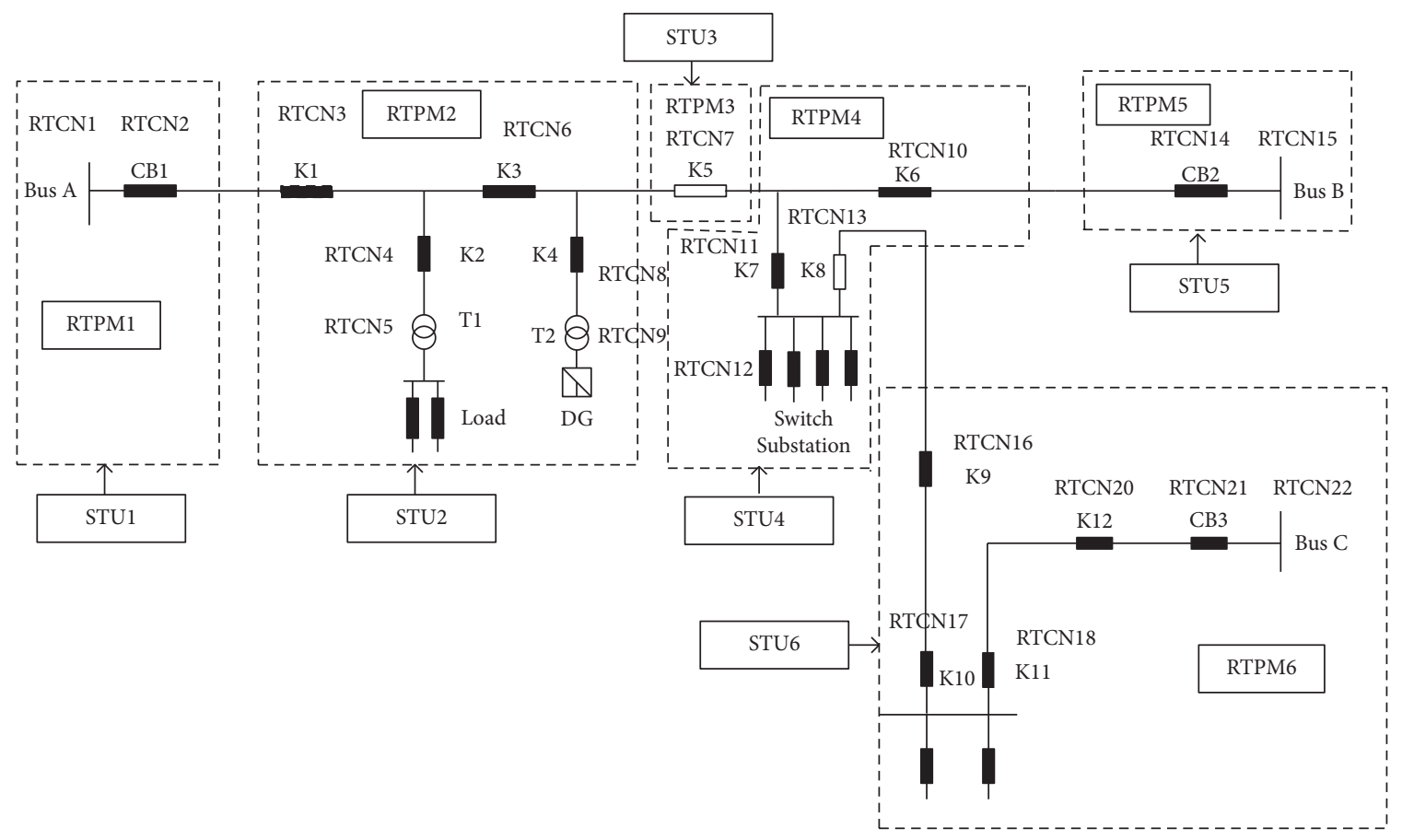

FIGURE 3: Schematic of distribution network topology.

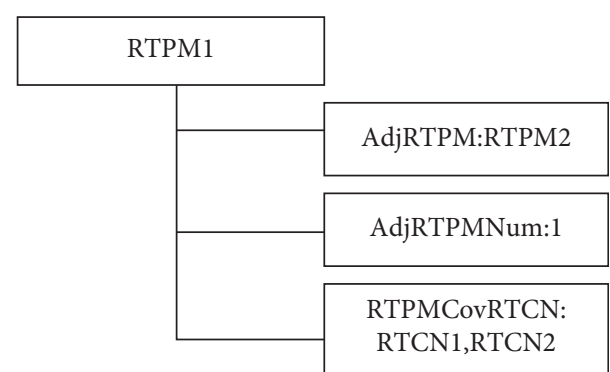

FIgure 4: Topological slice nodes RTPM1.

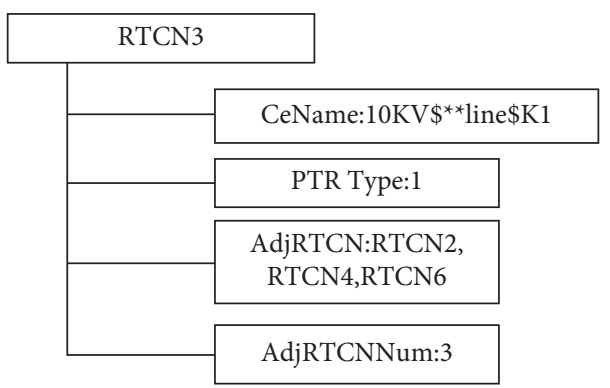

Figure 5: Logical node RTCN3. 


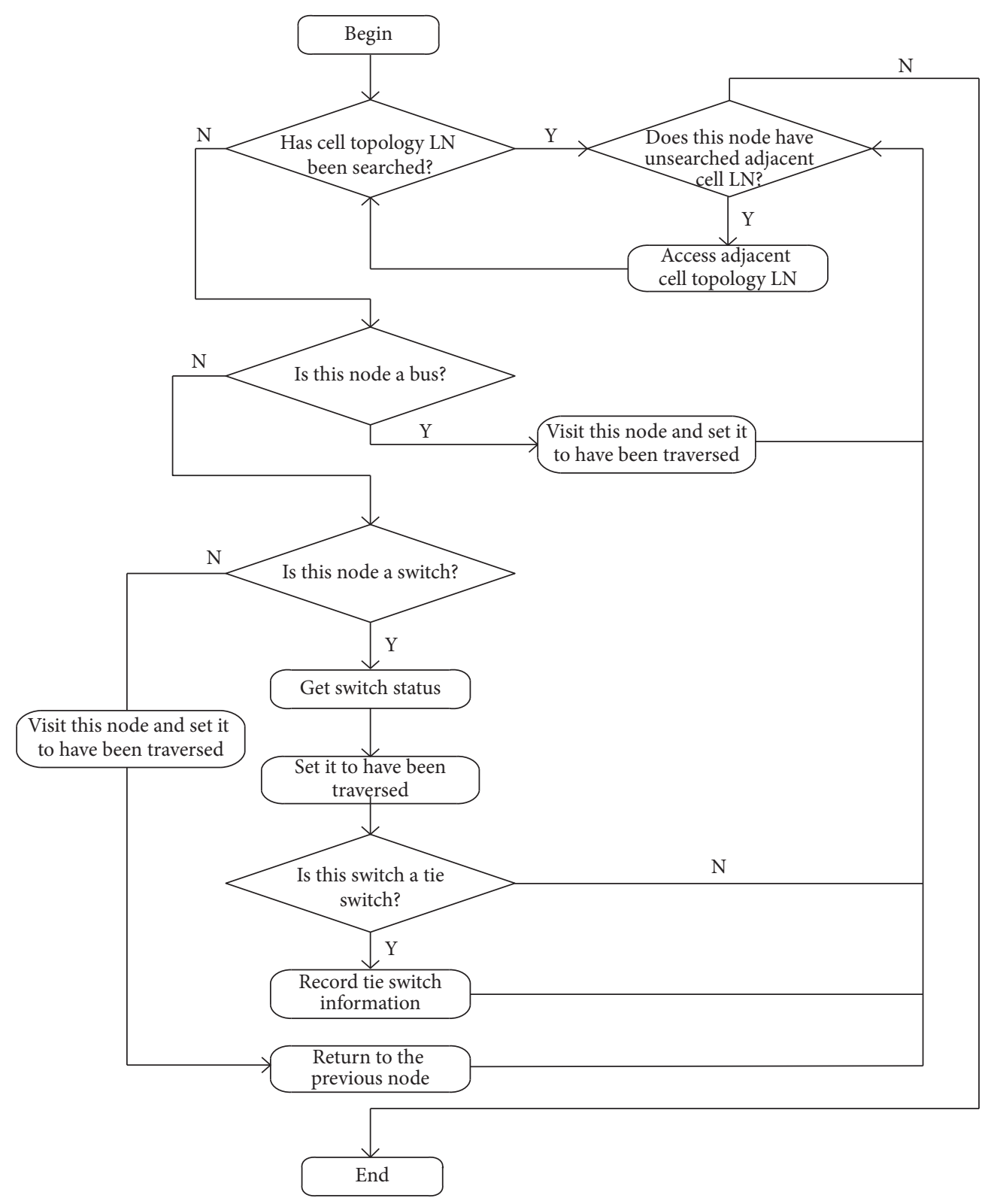

Figure 6: Algorithm flowchart.

\section{Topology Recognition Based on Topology LNs}

When equipment is installed or reduced in the distribution line, or the state of the tie switch changes, the corresponding STU notifies the distributed FA application master STU to initiate a topology search and update the application topology. Reading the local static topology configured in each STU and searching according to the current switch status can obtain the application topology. The GetDataValues service of IEC 61850 ACSI can complete the transmission of topology data.

Topology recognition is mainly two steps: first, search in the local topology configured by STU itself. When an adjacent cell topology LN is not in the current topology slice, the adjacent topology slice needs to be obtained. The STU initiates communication with the STU corresponding to the adjacent topology slice node, obtains the cell topology LNs of the adjacent topology slice, and then searches and connects multiple local area topologies to form a complete application topology of the feeder. The flow chart of the topology search algorithm is shown in Figure 6.

The process of connecting the logical nodes of the cell topology to form a topological slice is the process of identifying the local area topology of the line. In order to realize the real-time topological identification of lines, the first step is to traverse the graph. Starting from a given cell topology logical node in a connected graph, all cell topology logical nodes in the graph are accessed, and each cell topology logical node is accessed only once. There are two ways to traverse the connected graph: depth-first search (DFS) and breadth first search (BFS). In this paper, we use the static 
topology information stored in STU and the switch state on the feeder to identify the local area topology in the topology slice.

The feeder topology information represented by the cell topology LNs and the topology slice LNs is saved in the STU on the line, and each STU only stores the topology information related to the control domain of this STU. It is necessary to carry out real-time topology search across topology slices to transfer the local area topology information saved by STU. The complete topology search of feeders across the topology slice needs to select a master STU, and the master STU obtains the topology information saved by other STU to form the complete topology information of feeders.

The LNs of cell topology and depth-first algorithm are combined to search the feeder topology, and the bus is the starting point of topology search. Taking the distribution line shown in Figure 3 as an example, when the distribution network topology changes, STU1 is selected as the master STU to initiate the topology search. The search steps are as follows:

(1) Traverse from RTCN1, which represents bus A. Access RTCN1 and mark this node as traversed. The AdjRTCN element in RTCN1 points to RTCN2, a neighboring cell topology logical node that is not traversed.

(2) Access the cell topology logical node RTCN2, which represents the outgoing breaker CB1. Get the switch status of the outgoing breaker CB1, and mark this node as traversed. RTCN1 in the AdjRTCN element of RTCN2 has been traversed, while RTCN3 has not. There is no information of RTCN3 in the topology slice RTPM1, so STU1 communicates with STU2 corresponding to the adjacent topology slice RTPM2 to obtain the cell logic node information in the topology chip RTPM2, and carry out the next topology search across the topology slice.

(3) Access the cell topology logical node RTCN3. RTCN3 represents the switch K1, obtains the switch state of $\mathrm{K} 1$, and marks this node as traversed, and the topology search continues. RTCN2 in AdjRTCN element of RTCN3 has been traversed, while RTCN4 and RTCN6 have not. You can continue to search for RTCN4 and RTCN5 in this way.

(4) Access the cell topology logic node RTCN6. RTCN6 represents switch K3, obtains the switch state of K3, and marks this node as traversed. Switch K3 is a tie switch. RTCN3 and RTCN4 in the AdjRTCN element of RTCN6 have been traversed, while RTCN7 has not.

The search procedure of the cell topology logical node in RTPM3 is the same as the above steps. After the cross topology search is completed, the topology slices RTPM1, RTPM2, RTPM3, RTPM4, RTPM5, and RTPM6 are connected to form the whole feeder topology.
So far, the complete topology identification of feeders is completed. When the switch operates or the feeder structure changes, only the changed topology piece information needs to be updated to complete the real-time topology update.

\section{Summary}

Distributed control has perfect performance and fast response speed, which can effectively improve the safety of the distribution network and improve the power supply quality of the distribution network. Based on the IEC 61850 modeling technology, this paper builds new cell topology logical nodes and topology slice logical nodes based on the analysis of distributed application topology requirements and uses logical nodes to express the distribution line's topology. This method does not need to name each section of the line, simplifies the description of the topological structure, and can better support the realization of distributed control.

\section{Data Availability}

The data used to support the findings of this study are available from the corresponding author upon request.

\section{Conflicts of Interest}

The authors declare that they have no conflicts of interest.

\section{Acknowledgments}

The work was supported by the Key Project of Smart Grid Technology and Equipment of National Key Research and Development Plan of China (No. 2016YFB0900600) and Technology Projects of State Grid Corporation of China (No. 52094017000W)

\section{References}

[1] J. Zhou, L. Wang, M. Liu et al., "Research on Quick Distributed Feeder Automation for Fast Fault Isolation/SelfHealing in Distribution Network," in Proceedings of the 2019 IEEE Innovative Smart Grid Technologies - Asia (ISGT Asia), pp. 202-206, Chengdu, China, May 2019.

[2] Y. Li, "An Improved Fast Self-Healing Feeder Automation System," in Proceedings of the IEEE Advanced Information Technology, Electronic and Automation Control Conference, pp. 2428-2432, Xi'an, China, May 2018.

[3] L. Wang, J. Zhou, M. Liu et al., "Research on Application of New Intelligent Distributed Feeder Automation," in Proceedings of the 2019 IEEE 3rd International Electrical and Energy Conference (CIEEC), pp. 182-186, Beijing, China, September 2019.

[4] G. Zhabelova and V. Vyatkin, "Multiagent smart Grid automation architecture based on IEC 61850/61499 intelligent logical nodes," IEEE Transactions on Industrial Electronics, vol. 59, no. 5, pp. 2351-2362, 2012.

[5] N. Kashyap, C.-W. Yang, S. Sierla, and P. G. Flikkema, "Automated fault location and isolation in distribution grids with distributed control and unreliable communication," IEEE Transactions on Industrial Electronics, vol. 62, no. 4, pp. 2612-2619, 2015. 
[6] J. Liu, B. Yun, Q. Cui et al., "A distributed intelligent feeder automation system with fast self-healing performance," $A u$ tomation of Electric Power Systems, vol. 34, no. 10, pp. 62-66, 2010.

[7] Z. Zhu, Z. Jin, J. Chen et al., “Topology Self-Identification and Adaptive Operation Method of Distribution Network Protection and Self-Healing System," in Proceedings of the 2018 International Conference on Power System Technology (POWERCON), pp. 3087-3092, Guangzhou, China, November 2018.

[8] Z. Zhu, B. Xu, G. Han et al., "Study on the Feeder Topology Modeling and IED Configured Methods for IEC61850," in Proceedings of the 2014 China International Conference on Electricity Distribution (CICED), pp. 1482-1487, Shenzhen, China, September 2014.

[9] J. Ying, L. Chen, M. Liu et al., "Research on Check Method of Feeder Topology Model for Distribution Main Station," in Proceedings of the 2018 2nd IEEE Conference on Energy Internet and Energy System Integration (EI2), pp. 1-5, Beijing, China, October 2018.

[10] M. Zhang, P. Shen, W. Ji et al., "Feeder Topology Representation Method Based on Multiway Tree," in Proceedings of the 2016 IEEE International Conference on Power and Renewable Energy (ICPRE), pp. 451-455, Shanghai, China, October 2016.

[11] H. Liu, L. Mu, J. Su et al., "Centralized and intelligent control mode of feeder automation," Power System Technology, vol. 31, no. 23, pp. 17-21, 2007.

[12] R. Chen, J. Lu, M. Liu et al., "Distribution Network Topology Model Generation Method for Distributed Feeder Automation," in Proceedings of the 2019 IEEE Innovative Smart Grid Technologies - Asia (ISGT Asia), pp. 1057-1062, Chengdu, China, May 2019.

[13] G. Zhu, P. Shen, Y. Wang et al., "Dynamic identification method of feeder topology for distributed feeder automation based on topological slices," Power System Protection and Control, vol. 46, no. 14, pp. 152-157, 2018.

[14] W. Cong, Y. Zheng, Z. Zang et al., "Distributed storage and management method for topology information of smart distribution network," Automation of Electric Power Systems, vol. 41, no. 13, pp. 111-117, 2017.

[15] Z. Zhu, B. Xu, C. Guise, and G. Han, "Distributed topology processing solution for distributed controls in distribution automation systems," IET Generation, Transmission \& Distribution, vol. 11, no. 3, pp. 776-784, 2017.

[16] Z. Zhu, Key Technologies for the Application of IEC 61850 to Distributed Controls in Smart Distribution Grids, Shandong university, Jinan, China, 2018.

[17] K. Fan, B. Xu, J. Dong et al., "Identification method for feeder topology based on successive polling of smart terminal unit," Automation of Electric Power System, vol. 39, no. 11, pp. 180-186, 2015.

[18] Z. Zhu, B. Xu, Y. IP. Tony et al., "IEC 61850 based models for distributed feeder automation system," Automation of Electric Power Systems, vol. 42, no. 23, pp. 148-154, 2018. 